\title{
Ist die Zukunft eine Extrapolation?
}

\author{
VICTOR KRAFT
}

Herr P. Rohs hat meine Darlegung, daß die Zukunft eine Extrapolation aus dem bisher Erlebten ist, bestritten * - aber mit unstichhaltigen Argumenten.

Er will meine These dadurch widerlegen, daß bei ihrem Beweis die Zukunft schon vorausgesetzt werde: „Die Zukunft sei eine Extrapolation aus der Vergangenheit in die Zukunft".

Aber das ist eine völlig falsche Formulierung. Denn die Zukunft gibt es ja noch gar nicht vor der Extrapolation - also kann sie nicht extrapoliert werden und nicht eine Voraussetzung für die Extrapolation sein. Und es kann auch nicht die Zukunft extrapoliert werden, weil es sie noch nicht gibt. Mit der Zukunft wird nur die Fortsetzung des Geschehens, wie es in der Vergangenheit erlebt worden ist, extrapoliert als Fortsetzung des Geschehens über die Gegenwart hinaus. Die Extrapolation besteht darin, daß die bisher erlebte Fortsetzung des Geschehens darüber hinaus ausgedehnt wird. Damit wird aber ein neuer Bereich überhaupt erst geschaffen - eben die Zukunft.

Was mein Kritiker anstelle einer Extrapolation vertritt, ist der Kantische Gedanke: Die Behauptung der Zukunft sei (wie überhaupt die des Bestehens der Zeit) ein synthetisches Urteil a priori.

Wenn man diese Aussage als eine rein logische Charakterisierung versteht und als ein Urteil, das synthetisch ist und nicht aufgrund von Erfahrung gilt, kann man sie ohne weiteres anerkennen. Aber die negative Feststellung läßt die Frage offen: aufgrund wessen gilt es dann? Für eine Giltigkeit a priori muß der Grund aufgewiesen werden, sonst bleibt es eine dogmatische Behauptung. Welcher ist es? Die Extrapolation der Zukunft ist eine Annahme, die durch jedes neue Moment, in dem das Geschehen sich fortsetzt, bestätigt wird.

Die Zukunft ist eine notwendige Voraussetzung für GesetzmäBigkeit und GesetzmäBigkeit ist eine notwendige Voraussetzung für eine Erkenntnis, die mehr enthält als lediglich historisches Wissen. Das sind durchweg theoretische Notwendigkeiten. Wenn sie letztlich auf pragmatische Notwendigkeiten für das Leben zurückgehen, so betrifft das die Begründung der Erkenntnis überhaupt, wenn sie nicht durch eine Wesensschau platonischer Ideen in offener oder verschwiegener Weise gegeben werden soll (gegen S. 83 unten).

* Vgl. Peter Rohs, Ist die Zukunft eine Extrapolation? Bemerkungen zu einem Aufsatz von Victor Kraft, diese Zeitschrift, Bd. III, Heft 1 (1972), S. 81-84.

Adresse des Autors:

Dr. h.c. V. Kraft, em. Universitätsprofessor, A-1010 Wien I, Walfischgassa 15, Österreich. 